\title{
Pyrexia of Unknown Origin in Children in Singapore: A Twelve Year Experience
}

Lee EYX'1, Durnford $\mathrm{J}^{1}$, Rajan $\mathrm{C}^{1}$, Chong $\mathrm{CY}^{2}$, Thoon $\mathrm{KC}^{2}$, Li $\mathrm{JH}^{2}$, Arkachaisri T${ }^{3}$, Soh SY ${ }^{4}$, Tan $\mathrm{NWH}^{2}$

'Department of Paediatrics, KK Women's and Children's Hospital, Singapore

${ }^{2}$ Infectious Disease Service, Department of Paediatrics, KK Women's and Children's Hospital, Singapore

${ }^{3}$ Rheumatology and Immunology Service, Department of Paediatric Subspecialties, KK Women's and Children's Hospital, Singapore

${ }^{4}$ Haematology and Oncology Service, Department of Paediatric Subspecialties, KK Women's and Children's Hospital, Singapore

\begin{abstract}
Introduction
Pyrexia of unknown origin (PUO) is a diagnostic dilemma often faced in the pediatric setting. It is defined as daily fever more than 38.3 degrees Celsius for a minimum of 8 days, in whom no cause is apparent after initial outpatient or hospital evaluation that includes a careful history, physical examination and initial lab assessment.
\end{abstract}

\section{Methods}

We conduct a 12 year retrospective study of children admitted to KK Children's Hospital and first referred to the infectious disease (ID) service for PUO between December 2004 and May 2017, to determine the causes and outcomes of children referred for PUO.

Analysis was done using SPSS software version 20. Chi square test was used for categorical data, and Kruskal-Wallis test for continuous data. $\mathrm{P}$ values are considered statistically significant at $<0.05$.

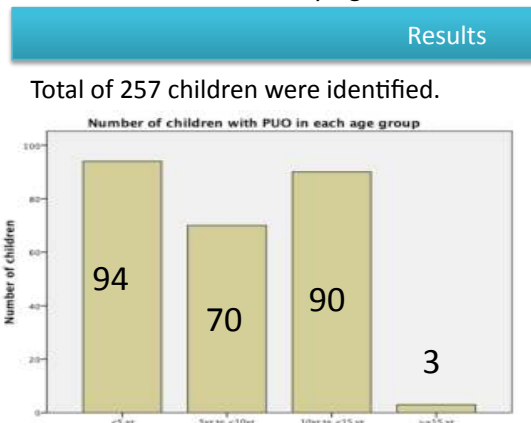

Fig, 1: Number of childrene with'PUO in each age group History and Physical examination

-Duration of fever before diagnosis of PUO was made: 8 days-14 months. Investigations

\begin{tabular}{|c|c|c|c|c|c|c|}
\hline Diagnostic Group & Infection & Inflammatory & Malignancy & $\begin{array}{l}\text { Otherss } \\
\text { Unnknown }\end{array}$ & HLH & \begin{tabular}{|c|c|} 
p value (chi square \\
test)
\end{tabular} \\
\hline Cough: $n(\%)$ & $73(53.7)$ & $20(39.2)$ & $2(18.2)$ & $17(32.7)$ & $3(42.9)$ & 0.024 \\
\hline Mouth ulcer: n $(\%)$ & $3(2.2)$ & $7(13.7)$ & $1(9.1)$ & $6(11.5)$ & $0(0)$ & 0.025 \\
\hline Doint pain: n $(\%)$ & $4(2.9)$ & $9(17.6)$ & $1(9.1)$ & $2(3.8)$ & $0(0)$ & 0.005 \\
\hline Lymphadenopathy: n(\%) & $26(19.1)$ & $30(58.8)$ & $4(36.4)$ & $13(25.0)$ & $4(45.1)$ & $<0.0001$ \\
\hline Hepatomegaly: n (\%) & $20(14.7)$ & $7(13.7)$ & $5(45.5)$ & $4(7.7)$ & $6(85.7)$ & $<0.0001$ \\
\hline plenomegaly: $n(\%)$ & $9(6.6)$ & $2(3.9)$ & 3 (27.3) & $1(1.9)$ & 5 (71.4) & $<0.0001$ \\
\hline
\end{tabular}

Table 1: Comparsion of symptoms and signs between the diagnostic groups for PUO

\begin{tabular}{|c|c|c|c|c|c|c|}
\hline Diagnostic Group & Infection & Inflammatory & Malignancy & Others/Unknown & Hнн & pvalue \\
\hline \begin{tabular}{|l} 
Highest WBC count, \\
$x 10 \%$ : \\
Median (IIRR) \\
\end{tabular} & $8.8(6.0-144.6)$ & $6.0(4.2-12.0)$ & $8.6(3.4-12.4)$ & $9.2(5.2-213.3)$ & $4.3(1.9-9.95)$ & 0.319 \\
\hline $\begin{array}{l}\text { lighest ANC count, } \\
\text { x10 9/: } \\
\text { Median (IIR) }\end{array}$ & $4.6(2.8-8.5)$ & $3.8(1.9-8.6)$ & $3.2(0.3-6.64)$ & $4.4(2.2-8.3)$ & $2.7(1.2-4.6)$ & 0.675 \\
\hline $\begin{array}{l}\text { Lowest PLT count, } \\
\text { x109,/L: } \\
\text { Median (IIRR) }\end{array}$ & $285.0(209.0-405.5)$ & $265.0(2120.0-414.0)$ & $\begin{array}{l}158.0 \\
(78.0-335.0)\end{array}$ & $\begin{array}{l}302.5 \\
(238.5-440.0) \\
\end{array}$ & $132.0(40.0-177.0)$ & 0.043 \\
\hline $\begin{array}{l}\text { Lowest Hb, g/DL: } \\
\text { Median (IQR) }\end{array}$ & $11.4(10.3-12.3)$ & $11.5(10.2-12.6)$ & $9.4(7.4-11.3)$ & $12.0(10.9-912.7)$ & $10.3(8.6-6-11.3)$ & 0.041 \\
\hline $\begin{array}{l}\text { Highest CRP, mg/L: } \\
\text { Median (IIRR) }\end{array}$ & 43.4(7.9.-106.8) & $34.3(16.3-100.5)$ & $51.8(36.8-96.2)$ & 23.9(7.2-63.0) & 78.6 (770.9-231.9) & 0.018 \\
\hline $\begin{array}{l}\text { Highest ESR, } m \mathrm{~m} / \mathrm{hr} \text { : } \\
\text { Median (IIRR) }\end{array}$ & $60.0(28.0-95.0)$ & $63.0(37.0 .90 .0)$ & $82.0(30.0-123.0)$ & $39.0(24.0-79.0)$ & 80.0 (26.0-105.0) & 0.349 \\
\hline $\begin{array}{l}\text { Highest ALT, U/L: } \\
\text { Median (IQR) }\end{array}$ & $26.0(15.0-53.0)$ & $20.5(16.0-47.3)$ & $36.0(11.0-61.0)$ & $21.0(14.0-40.3)$ & $103.0(50.3-248.8)$ & 0.099 \\
\hline $\begin{array}{l}\text { Highest AST, U/L: } \\
\text { Median (IRR) }\end{array}$ & $38.0(26.0-62.0)$ & $35.5(28.8-52.5)$ & $45.0(35.0-63.0)$ & $38.5(26.0-50.3)$ & $153.5(49.0-758.5)$ & 0.084 \\
\hline $\begin{array}{l}\text { Highest LDH, U/L: } \\
\text { Median (IIR) }\end{array}$ & $504.0(287.0-844.0)$ & $586.0(3977.5-832.5)$ & \begin{tabular}{|l|}
$\begin{array}{l}451.0 \\
(174.5-1207.0)\end{array}$ \\
\end{tabular} & \begin{tabular}{|l}
530.5 \\
$(308.0-806.3)$ \\
\end{tabular} & 764.5 (4811.3-6482.5) & 0.22 \\
\hline
\end{tabular}

SingHealth $A$

Sing Health Academic Healthcare Cluste

[1] Chow et al. Fever of unknown origin in children: A systematic review. World Journal of Paediatrics February 2011; 7 (1); 5-10

Thingarere 10 and 109 girls $(42.4 \%)$ -Race: Chinese $47.5 \%$ $17.1 \%$, Others $13.2 \%$ -Age: ranges from 2 months 192 months, median 81 months (IQR 36.5-132 months). Figure 1 shows the age distribution of children. higher CRP.

Diagnoses

Treatment and Outcomes

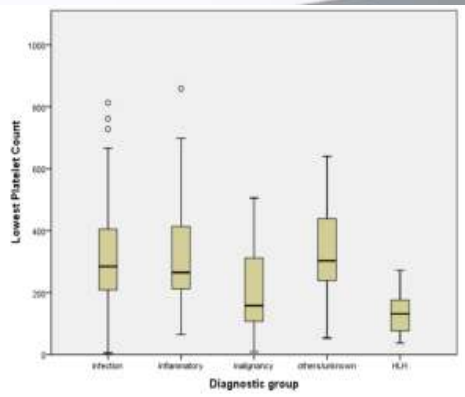

Fig. 2: Boxplot comparisons of Lowest platelet counts between the diagnostic groups for PUO

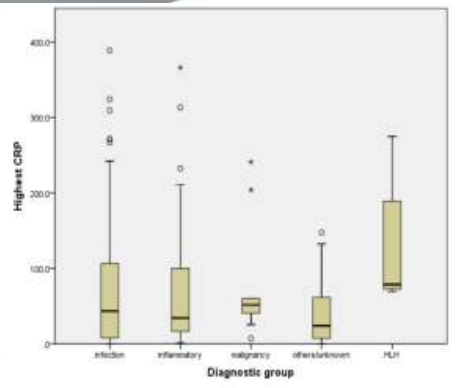

Fig. 3: Boxplot comparisons of Highest C-reactive Protein levels between the diagnostic groups for PUO

Table 1 shows the comparison of the symptoms and signs between the various diagnostic groups for PUO. Table 2 shows the comparison of blood results. Figures $2 \& 3$ show the boxplot comparisons of lowest platelet counts and highest CRP levels between the various diagnostic groups for PUO respectively. As illustrated by Table 1 and 2 and Figures 2 and 3, patients with Haemophagocytic lymphohistiocytosis (HLH) are more likely to have hepatomegaly, splenomegaly, and have lower platelet counts and

Table 3 shows the causes of PUO identified. Infective causes accounted for $52.9 \%$ of PUO cases, followed by inflammatory causes (19.8\%), malignancy (4.3\%), HLH (2.7\%), others (0.8\%).

\begin{tabular}{|c|c|c|c|c|c|}
\hline cous & Ereavence & canse & freaned & chuse & Ereuend \\
\hline infectrove conser & $136(5225)$ & Intertive caves I Conets & $236(32.969)$ & Mategrancy/Haematology & 13(4.35) \\
\hline Inthoid & 19 & manaras & 1 & Lentamia & 4 \\
\hline Mrcoplisma infection & 12 & MaN1 virus infection & 1 & Lnmphoma & 2 \\
\hline 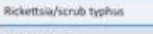 & 12 & 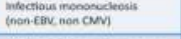 & 1 & Luer embryonal sartoma & 1 \\
\hline Thabecadsias" & 12 & 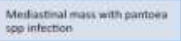 & 3 & Neurabiastoma & 1 \\
\hline 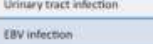 & 10 & Meiodans & 1 & Adreera gland hientr & 1 \\
\hline Sinuits & $\cdot$ & 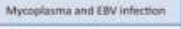 & 1 & unmor & \\
\hline 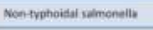 & 6 & ocesut bacteremena & 1 & 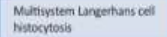 & 1 \\
\hline nevenons: & 4 & Statropharnessal absess & $\frac{1}{1}$ & Severe aplustic nemu & 1 \\
\hline Hevinterison & 3 & 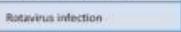 & 1 & & \\
\hline Otrsa mesia & 3 & Tuberatoid suachacitary & 1 & Othens & $2(0.8 \times)$ \\
\hline 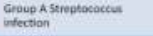 & 3 & leprosy & & Evanissndrome & 1 \\
\hline inective endocarditis & 3 & & & 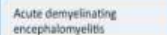 & 1 \\
\hline Parainfluence 3 vira & , & innummetory caves & $51(19.25)$ & & \\
\hline Bscteral enterocolitus & 2 & Ohachi ithesese. & 25 & Unknown & $50(19.5 \times)$ \\
\hline Covvinfection & 2 & Hawstifu deseare & $\mathrm{n}$ & & \\
\hline 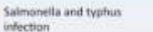 & 2 & SIE & 4 & нен & $7(2.7 \times)$ \\
\hline Oivemingnesece infertion & & Kawaral atseose & 3 & & \\
\hline Oiseminoted bece infertion & 2 & inflaminatorn bowel dseose & 2 & \multirow{6}{*}{\multicolumn{2}{|c|}{$\begin{array}{l}* 20 \text { children had biopsy-proven } \\
\text { Kikuchi disease. } \\
* * 5 \text { children had positive AFB } \\
\text { cultures for Tuberculosis. } \\
* * * \text { Immune dysregulation- } \\
\text { Evans-type (STAT3 mutation) }\end{array}$}} \\
\hline Enterovinut intection & 2 & 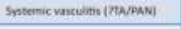 & 1 & & \\
\hline Adenorins and tinnosers & 1 & 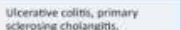 & 1 & & \\
\hline RST and enterowives & 1 & 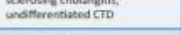 & & & \\
\hline RSS infectoon & 1 & Detess sporomere & 1 & & \\
\hline Adenovirum & 1 & 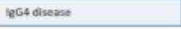 & 1 & & \\
\hline
\end{tabular}

Table 3: Causes of PUO in the study children

A similar Canadian study by Chow et al showed that the commonest causes for PUO were infection for 832 (51\%), collagen vascular disease for 150 (9\%), malignancy for $93(6 \%)$, miscellaneous non-infectious conditions for 179 (11\%) and no diagnosis in 384 (23\%) children.[1]

Most children had resolution of fever with treatment of underlying disease and were discharged well. Those with unknown cause of fever had a selflimited course of their illness. Death occurred in 8 children.

\section{Conclusion}

PUO in children was mostly due to infective or inflammatory causes. Attention to uncovering exposures, symptoms, signs or laboratory findings is the most fruitful approach to finding the cause of PUO. PATIENTS. AT THE HE RT OF ALL WE DO. 